\title{
EPIGENESIS AND DYNAMIC SIMILARITY IN TWO REGULATORY NETWORKS IN PSEUDOMONAS AERUGINOSA
}

\author{
Janine F. Guespin-Michel ${ }^{1}$, Gilles Bernot ${ }^{2}$, \\ Jean Paul Comet ${ }^{2}$, Annabelle Mérieau ${ }^{1}$, Adrien Richard ${ }^{2}$, \\ Christian Hulen ${ }^{3}$ and Benoit Polack ${ }^{4}$
}

${ }^{1}$ Laboratoire de microbiologie du froid, EA 2123, Université de Rouen, F-76 821 Mt St Aignan, France. Email: janine.guespin@univ-rouen.fr (corresponding author).

${ }^{2}$ LaMI, CNRS UMR 8042, Université d'Évry-Val-d'Essonne, Boulevard François Mitterrand, 91025 Evry Cedex, France.

${ }^{3}$ Université de Cergy-Pontoise UFR sciences et techniques St Martin, 2 av A. Chauvin, BP 222, 95300 Pontoise, France.

${ }^{4}$ GREPI EA 2938 Université Joseph Fourier Grenoble 1, Laboratoire d'Hématologie, CHU de Grenoble, BP 217, 38043 Grenoble cedex 9, France.

\begin{abstract}
Mucoidy and cytotoxicity arise from two independent modifications of the phenotype of the bacterium Pseudomonas aeruginosa that contribute to the mortality and morbidity of cystic fibrosis. We show that, even though the transcriptional regulatory networks controlling both processes are quite different from a molecular or mechanistic point of view, they may be identical from a dynamic point of view: epigenesis may in both cases be the cause of the acquisition of these new phenotypes. This was highlighted by the identity of formal graphs modelling these networks. A mathematical framework based on formal methods from computer science was defined and implemented with a software environment. It allows an easy and rigorous validation and certification of these models and of the experimental methods that can be proposed to falsify or validate the underlying hypothesis.
\end{abstract}

\section{INTRODUCTION}

The word epigenesis was first coined by Harvey in 1651, to describe the gradual formation of the different parts of an embryo. However, this word took on a new meaning when geneticists developed the notions of genotype and phenotype. Epigenetic modifications arise and can be transmitted from a cell to its progeny in the absence of genetic or environmental modifications. They may be triggered by an environmental signal but remain upon disappearance of this signal. Thus, several stable phenotypes may arise from the same genome in the same conditions.

This is the biological equivalent of the physicist's description of multiple steady states arising in non-linear dynamic systems. It has been shown in several instances that multistationarity is a good description of biological processes such as epigenesis (Guespin-Michel et al., 2003), differentiation and memory (Delbrück, 1949; Demongeot, 1998; Casadesus and D’Ari, 2002). Thomas (1981) conjectured that a 
positive feedback circuit is a necessary condition for the presence of multiple steady states in a dynamic, non-linear system, and this was formally verified later (Snoussi, 1998; Cinquin and Demongeot, 2000; Soulé, 2003). This means that considering the presence and the nature of feedback circuits may lead to useful hypotheses concerning the behaviour of a biological system (Thomas and Thieffry, 1995; Thomas, 1998). Feedback circuits often involve several levels of molecular interactions (transcription, translation, metabolic interactions, etc.). However, transcriptional regulatory networks may display such circuits, generally embedded in larger systems. It is then possible to extract simple models from these networks, that only take into account the interactions responsible for feedback (Thomas and Kaufman, 2001; Kaufman and Thomas, 2003).

In a preceding workshop of the SFBT (2000) this led us to propose that the production of mucus by strains of Pseudomonas aeruginosa isolated from the lungs of patients suffering from cystic fibrosis may be due to an epigenetic modification. A model was also proposed to help design experiments to test this proposal (GuespinMichel and Kaufman, 2001). Here we show that the very same abstract model can be proposed for an otherwise quite different regulatory network controlling the acquisition of cytotoxicity in the same bacterium in similar conditions, highlighting the possible dynamic similarity between these regulatory networks.

Starting from these biological examples we have created a new software environment for these abstract regulatory networks, based on the aforementioned theorem of R. Thomas, which integrates sophisticated algorithms from computer science (SMbioNet). This software environment provides biologists with a model validation tool and provides a rigorous basis for the concept of dynamic similarity.

\section{MUCOIDY}

The opportunistic bacterium, $P$. aeruginosa, is the major cause of mortality in cystic fibrosis. In the lungs of cystic fibrosis patients but in no other growth conditions, P. aeruginosa becomes able to produce a heavy mucus (alginate) that enhances its antibiotic resistance and increases the respiration deficiency of the patient (Govan and Harris, 1986). Bacteria isolated from the lungs of such patients can be cultivated and form more or less stable mucous colonies called mucoid variants. The regulatory network controlling alginate synthesis has been extensively studied (Schurr et al., 1994). The main regulator was shown to be a sigma factor, AlgU, which is necessary for the expression of several operons involved in alginate production and regulation, including its own operon, the algU mисABCD operon. muc genes encode proteins that form an anti-AlgU membranous complex (Rowen and Deretic, 2000) (Figure 1a).

This leads to the interlocking of a positive feedback circuit (AlgU is necessary for its own synthesis) and a negative feedback circuit (AlgU is necessary for the synthesis of its inhibitors). Therefore, the conditions required for epigenesis are met. However, since a mutation has been identified in the $m u c A$ gene of many mucoid strains (Boucher et al., 1997), it is generally admitted that this mutation is responsible for the acquisition of the mucoid phenotype. By contrast, we have proposed that the presence of bacteria in the patient's lungs induces an epigenetic modification (synthesis of high amount of $\mathrm{AlgU}$, leading to the stable production of mucus) (Guespin-Michel and Kaufman, 2001). In our hypothesis, the antisigma factor, a membrane complex that 
traps $\mathrm{AlgU}$, must be produced, together with $\mathrm{AlgU}$ protein itself, at a higher rate than in the non-mucoid strains, which may constitute a high metabolic burden. The epigenetic modification to mucoidy might thus, in turn, prompt the selection of mutants impaired in the membrane complex. We claim that in the patient's lungs, an epigenetic modification may precede the selection of mutations.

a)

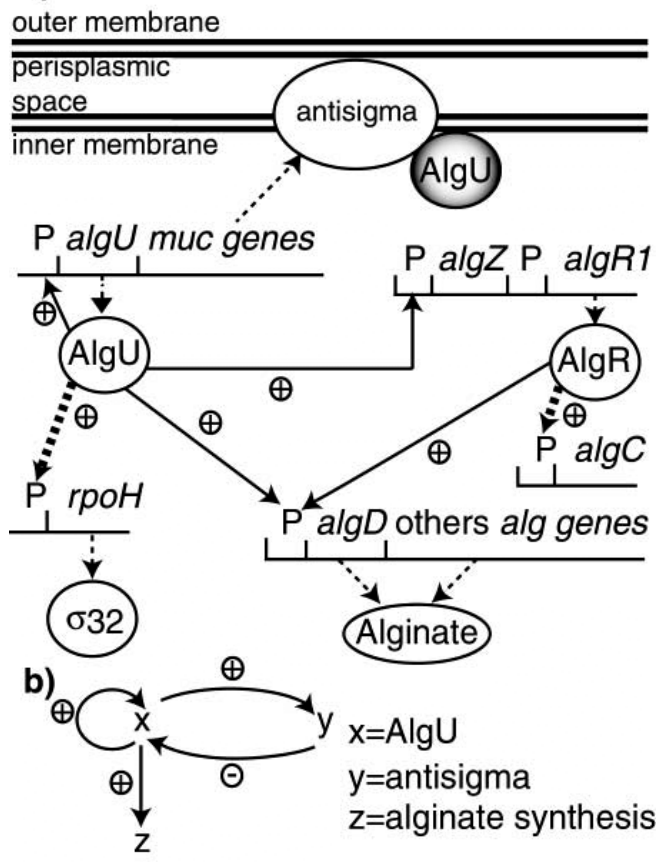

Figure 1. Regulatory network for alginate synthesis in P.aeruginosa. a) Schematic molecular diagram featuring the main steps of the regulation. The $\mathrm{Alg} \mathrm{U}$ protein is a sigma factor that is essential for the transcription of its own operon, and for the transcription of all the operons involved in the regulation and the production of alginate. The $\operatorname{alg} U$ operon comprises four other genes (muc), three of which are involved in the inhibition of protein $\mathrm{AlgU}$ by forming a complex which inserts in the membrane ands traps protein AlgU out of its DNA targets. Heavy dotted arrows are not considered in the formal graph. b) Formal graph. $X=\mathrm{AlgU}$, $Y=$ the algU inhibitor; $Z=$ the response, leading to alginate synthesis.

This epigenetic hypothesis has been first tested by modelling (Guespin-Michel and Kaufman, 2001). A very simplified model of the regulatory network has been constructed as depicted in Figure $1 \mathrm{~b}$. The three variables are $x=\mathrm{Alg} U, y=$ the $\mathrm{AlgU}$ inhibitors and $z=$ alginate production. The four edges represent: the autoregulation of variable $x(x \rightarrow x)$, the transcription of the genes encoding the antisigma factors $(x \rightarrow y)$, the transcription of the genes involved in alginate production $(x \rightarrow z)$, and finally the inhibition of AlgU by the antisigma factors $(y \rightarrow x)$. Two feedback circuits control $\mathrm{AlgU}$, a positive feedback circuit at the transcriptional level, and a negative feedback circuit involving the activity of the AlgU sigma factor.

This model has been first studied by generalised logical analysis (Snoussi, 1989; Thomas and D'Ari, 1990). This showed that the epigenetic hypothesis (the possibility that two stable states may exist depending on the previous history of the system) is coherent and that biologically consistent values of the parameters in the equation can lead to properties that mimic those of the system. However, there is more to it. The model predicts that a pulse of $\mathrm{AlgU}$ (provided by placing the $\operatorname{alg} U$ gene under the 
control of an inducible promoter) will suffice to switch wild type bacteria to a mucoid state. The model is thus predictive as well as explanatory.

\section{CYTOTOXICITY}

In order to become mucoid in cystic fibrosis lungs, the bacteria must first multiply to a rather high density, which should be prevented by the host's defences. However, many mucoid bacteria isolated from the patient's lungs are also cytotoxic and therefore they are able to lyse the host's macrophages and thus overcome these defences (Dacheux et al., 2000, 2001a). Cytotoxic P. aeruginosa, like many other cytotoxic bacteria synthesise a secretory apparatus (Type III) that enables them to inject toxins from their cytoplasm into the target cell (or in the medium when the contact is simulated by laboratory conditions such as Calcium depletion; Feltman et al., 2001; Franck, 1997). By contrast, most $P$. aeruginosa strains from the environment are not able to become cytotoxic in these conditions. Only bacteria isolated from patients suffering from diseases such as cystic fibrosis have this ability which they retain more or less stably when plated on agar medium. No mutation responsible for this ability has been reported; indeed, the nature of the mechanism which leads to this ability is not generally addressed in the literature. It is therefore interesting to investigate whether it could be epigenetic.

It must be noted that there is no strict correlation between mucoidy and cytotoxicity since bacteria isolated from the patient's lungs can display both phenotypes or only one of them (Banwart et al., 2002; Dacheux et al., 2001a; Moss et al., 2001). The regulatory networks responsible for the production and regulation of the proteins involved in Type III secretion have been studied in several bacterial pathogens (Hueck, 1998) including recently P. aeruginosa (Franck, 1997). Cytotoxicity is controlled by a main regulator, ExsA, which positively regulates in trans the expression of all the operons involved in the process, including its own, namely the production of the secretory apparatus and toxins (Dacheux et al., 2001b). The first gene exs $D$ of one of the operons regulated by ExsA, encodes an inhibitor of ExsA. (McCaw et al., 2002) (Figure 2a). Two feedback circuits thus control ExsA, a positive feedback circuit at the transcriptional level and a negative feedback circuit involving the activity of ExsA. These circuits are interlocked since ExsA is necessary for both its own synthesis (the positive feedback circuit) and the synthesis of its inhibitor (the negative feedback circuit). Thus a minimal formal graph can be drawn that is strictly identical to that for the regulatory network of mucus production (Figure 2b). The three variables are $x=$ ExsA, $y=$ the ExsA inhibitor ExsD, and $z=$ type III secretory apparatus and toxin production. The four edges represent: the autoregulation of variable $x(x \rightarrow x)$, the transcription of gene $\operatorname{exs} D(x \rightarrow y)$, the transcription of the genes involved in type III secretory system $(x \rightarrow z)$, and finally the inhibition of ExsA by ExsD $(y \rightarrow x)$. Therefore, the hypothesis of an epigenetic modification can be proposed for the same reasons as for the mucoid phenotype. Since the model in Figure $2 b$ is strictly identical to that of Figure $1 b$ and since the hypothesis to be tested is the same (epigenesis), the results in terms of epigenesis are the same and the same type of experiment can be proposed to test the hypothesis. This holds true even though: i) other genes are involved in the cytotoxic regulatory network and 
their precise role is unknown although it does not seem to involve another circuit (Dacheux et al., 2002); ii) this description does not take into account the inducing role of the presence of the target cell (or of calcium depletion in laboratory conditions); iii) the description of the regulatory network is limited to transcription and does not contain the regulatory role that is presumably exerted at the level of the secretory apparatus itself (Miller, 2002).

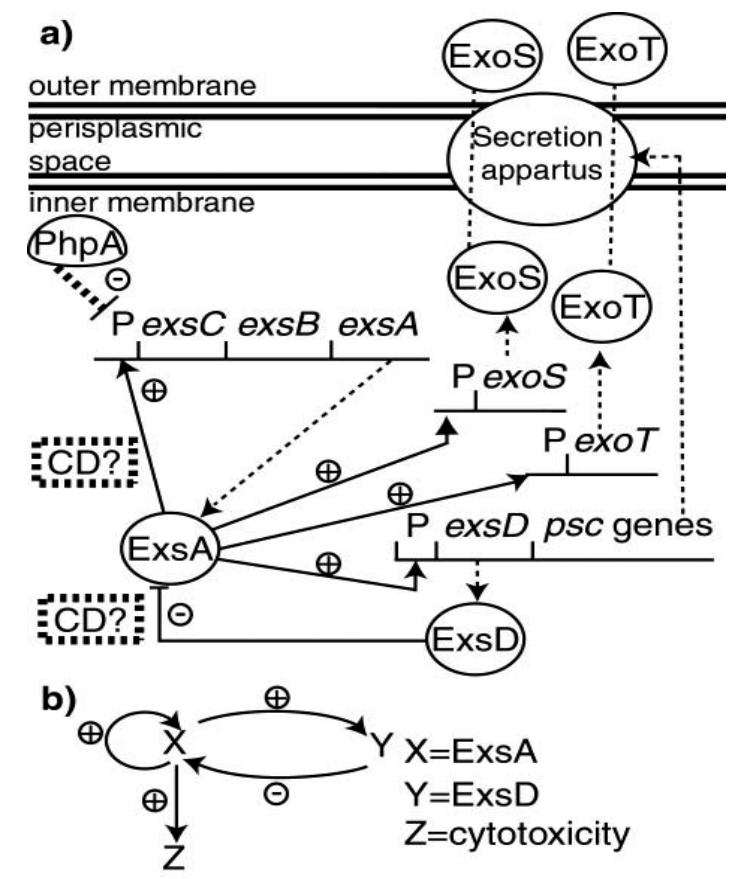

Figure 2. Regulatory network for cytotoxicity. a) Schematic molecular diagram featuring the main steps of the regulation. The main postive regulator ExsA controls all the operons involved in cytotoxycity including its own operon and the operon containing the gene for the ExsA inhibitor, ExsD. Heavy dotted arrow and frames are not considered in the formal diagram. The target for Calcium depletion (CD) is not completely elucidated up to now, and two hypotheses are considered here. b) Formal graph. $X=$ ExsA, $Y=\operatorname{ExsD} ; Z=$ the response, leading to cytotoxicity.

\section{FORMAL LOGIC TO PROPOSE MODELS AND EXPERIMENTS}

\section{Temporal Logic and Model checking}

The mathematical treatment of this model has been presented (Guespin-Michel and Kaufman, 2001) but a formal treatment can also be performed on a computer provided that the properties are formally defined. The objective of such a formalisation of the mathematical models is to make it possible to exhaustively study all the sensible models; this in turn facilitates the design of experiments to validate or invalidate them (Bernot et al., 2002, 2003a). Behavioural properties where time plays a central role have to be formalised, thus we use temporal logics (Huth and Ryan, 2000).

For a given formal graph, there is a large number of dynamic models depending on the values of thresholds and parameters. Each one defines a specific temporal behaviour. Experimental knowledge can be expressed using several temporal logic formulae. Each temporal logic formula expresses a property which can be used to split the set of possible dynamic models into two parts: those which satisfy the property and 
those which do not. The splitting can be computed automatically using the Model Checking algorithm. Consequently, formalising biological knowledge into temporal formulae allows us to automatically extract the sensible models (Pérès and Comet, 2003).

Our software environment SmbioNet allows the user to draw the regulatory network as in Figures $1 \mathrm{~b}$ and $2 \mathrm{~b}$, and generate all the compatible models. It then permits the user to enter temporal logic formulae and to keep only the models which satisfy them (Richard et al., 2000). Entered in SMbioNet the two graphs generate 648 dynamic models, each one with its own behaviour, corresponding to different thresholds and parameters.

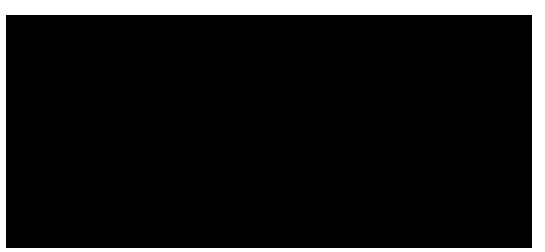

b)

\begin{tabular}{|c|c|l|l|}
\hline$x$ & $y$ & $K x$ & $K y$ \\
\hline 0 & 0 & $K x\{y\}=0$ & $K y\{\}=0$ \\
\hline 0 & 1 & $K x\{\}=0$ & $K y\{\}=0$ \\
\hline 1 & 0 & $K x\{x y\}=2$ & $K y\{\}=0$ \\
\hline 1 & 1 & $K x\{x\}=1$ & $K y\{\}=0$ \\
\hline 2 & 0 & $K x\{x y\}=2$ & $K y\{x\}=1$ \\
\hline 2 & 1 & $K x\{x\}=1$ & $K y\{x\}=1$ \\
\hline
\end{tabular}

c)

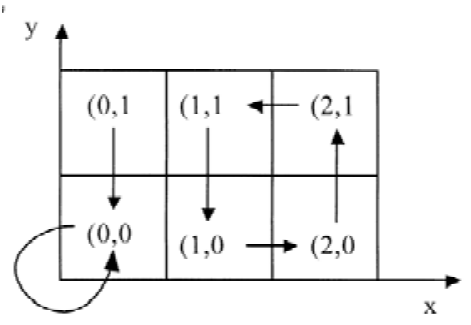

Figure 3. A Model satisfying the epigenetic hypothesis. a) Formal graph. The threshold of the regulation of $x$ on itself is inferior to that of the regulation of $x$ on $y$. Since $z$ is not a regulator, it does not have any influence on the dynamic and thus it is not represented. b) Parameter table. The two first columns define the states of the network and the two others the parameters. A parameter $K x, w$ gives the expression level towards which $X$ tends to evolve when $w$ represents all the positive regulatory effects that are active on $X$ (including the lack of active negative effects). For the given example, $w$ reflects the effects of $x$, if it's value is higher than the activity thresholds indicated in the graph, and the effects of $y$, if its value is lower than the activity threshold. c) State transition graph. The dynamics of the model is directly deduced from the values of the parameters (see Bernot et al. (2003b, 2004) for details).

\section{Consistency of the epigenetic hypothesis}

The epigenetic hypothesis means that even in the presence of the inhibitor $(y)$ it is possible to have a recurrent state in which mucus production (or cytotoxicity) ( $z$ ) is activated. The epigenetic hypothesis is consistent if and only if there exists at least one model with the $(y \rightarrow x)$ inhibition such that the behaviour can reach a state from which $z$ is activated in a recurrent way. This amounts to verifying if there is a model such 
that, if at a given time the bacterium has acquired the new phenotype (mucoidy or cytotoxicity), then later it will be again in the same state. This is formally expressed according to temporal logic as:

$$
(z=1 \rightarrow \text { Fs }(z=1)), \text { where Fs means "in a strict future". }
$$

Since the non-mucoidy (resp. non-cytotoxicity) phenotype is stable in the wild bacterium, the models to be considered for consistency must also have a behaviour where a non-mucoid bacterium (resp. cytotoxic) cannot become mucoid (resp. cytotoxic) later on. This is formally expressed as:

$$
(z=0) \rightarrow \text { always }(z=0) .
$$

By giving these formulae to SMbioNet we can automatically know how many models (and which ones) satisfy the formulae. Since $z=1$ requires that $x=2$, the epigenetic hypothesis means that the relative forces of the two circuits are such that it is possible to make recurrent $x=2$. Consequently the previous formulae are equivalent to $[(x=2 \rightarrow$ Fs $(x=2))$ and $(x=0 \rightarrow$ always $(x<2))]$ as well as to $[(x=2 \rightarrow$ Fs $(z=1))$ and $(x=0 \rightarrow$ always $(z=0))]$. SMBioNet has shown that eight models satisfy these formulae (Richard et al., 2000; Bernot et al., 2003b, 2004), two of which ensure that all the regulations of the network are active (these two models cannot be deduced from simplest networks). Figure 3 gives one of these two models: when $(x=0)$ then we always have $(x=0)$, thus it is not possible to evolve towards a state where $(x=2)$. When $(x=2)$ the state of the network is in a cycle from which one cannot exit and in which $(x=2)$ periodically.

\section{Experimental proof design}

The consistency of the epigenetic hypothesis being established, the next step is to propose experiments which prove it in vivo. The second formula is obviously satisfied in vivo. Thus only the first formula has to be tested. As we can see in Table 1, when the left part of the formula $(x=2 \rightarrow$ Fs $(z=1)$ ), is false, the formula is always true regardless the value of the right part. So the only relevant experiments start by assigning (artificially if necessary) $x$ to 2 and the whole formula is true if and only if Fs $(z=1)$ is true, that is if and only if after a lapse of time $z$ is equal to 1 . The scenario of experiments is thus the following:

1. Start by pulsing $x$ to saturation with an external signal until it imposes that $(x=2)$ and then stop the pulse.

2. Wait a lapse of time to allow the system to settle down and observe the phenotype. The length of this "lapse of time" must be determined experimentally; here it stands for "as many subsequent generations as possible." If the bacterium has not changed its phenotype, then the experiment a priori fails. If the bacterium has acquired a new phenotype (mucous or cytotoxic), that lasts at least several generations after the external signal has been removed, then, epigenesis is proven.

Of course, this is only useful if this prediction is amenable to experimentation, (it is "operable"), and if the results of the experiments are "observable". In other words, is it possible to use some device to raise $x$ to 2 , then remove this device, and then observe the modification of the phenotype at different times? 
In the case of $P$. aeruginosa the first requirement involves the possibility of increasing $x$ temporarily without introducing the bacteria into the lungs of a cystic fibrosis patient. Several methods can be used of which a pulse of protein " $x$ " from a genetically engineered construction is a standard method.

Table 1. Truth table of the logical connector $p \rightarrow q$

$p \rightarrow q$ is true:

1. When $p$ is false, the implication is true whether $q$ is false or true.

\begin{tabular}{|l|l|l|}
\hline$p \quad \backslash \quad q$ & true & false \\
\hline true & true & true \\
\hline false & false & true \\
\hline
\end{tabular}

2. When $p$ is true AND $q$ is true;

Observing the results of this experiment may be less straightforward. We have considered $z$ as being the output of the model and have designated it as "the phenotype". However, the model is largely confined to the transcriptional level, so strictly speaking, $z$ represents the expression of the genes leading to the phenotype, i.e. genes of the alginate synthesis operon, or genes of Type III apparatus and toxins respectively. It may be that gene expression is indeed an epigenetic mechanism whereas the phenotype itself is not, due to another level of regulation.

\section{Limits of the approach}

The grounding of the model in the transcriptional network alone highlights one of the limits of this approach. Other limits are illustrated by the two chosen cases. In both cases the formal graph on which the models are based is actually only a subgraph of a more general graph showing the regulatory network (Figures 1a and 2a, are simplified with regard to present knowledge but they are more complicated than the part represented in Figures $1 \mathrm{~b}$ and $2 \mathrm{~b}$ ). What would be the consequences of considering all the interactions? Having neglected the outgoing edges of the graph, (Figure 1a) does not have any consequence since we are only interested in the subsystem involving the production of mucus. On the other hand, our neglecting the edges entering this subsystem (Figure 2a) might have an important impact. By construction of the graph, some situations can be eliminated.

1) By construction, we call $z$ the "response" of the regulatory network, and gather into one edge, all the edges that correspond to the effect of AlgU (resp. ExsA), directly or indirectly on the production of mucus (resp. exotoxins).

2) At the present state of our knowledge, all genes which are not included in our model and have a direct influence on $x$ or $y$ do not participate in a functional feedback circuit. According to Snoussi (1989) this results in the number of steady states not being affected. Thus multistationarity is preserved.

Let us remark that the formal dynamics of the system, which is submitted to the model checker, only consider the influence of the variables explicitly mentioned in the regulatory network. Thus, it models in a certain sense a "closed system" where variations of the environment are not taken into account. In practice, it means that genes or external signals, which can vary in time although they are not involved in a feedback circuit, are ignored. Consequently our work remains valid a priori only if neglected genes have a constant influence on the variables. We believe that such 
artificially simplified situations nevertheless give valuable information but it stands to reason that only in vivo experiments can tell us if the choice of the genes considered as relevant for the model is sensible. This is for example the case of the cytotoxic phenotype, where calcium depletion variations may have a non constant influence on $x$ (the molecular mechanism is yet under scrutiny). The study presented here makes these influences artificially negligible by taking into account only a situation of constant calcium depletion.

\section{CONCLUSION}

The availability of high throughput data has made it possible to compare and classify large amounts of data. Transcriptional regulatory networks constitute one important way of ordering these data so as to understand the functioning of living organisms, providing one does not underestimate the fact that different levels of regulation often intermingle (Alm and Arkin, 2003). A few methods are available for studying transcriptional regulatory networks. Looking for homologies in gene or protein data bases is the most common one and can give useful hints about functions. One can also look for the operation of similar mechanisms such as the role of sigma factors, upstream regulators, and the supercoiling or bending of the promoter and upstream regions in bacteria.

A new method has been proposed that extracts the most frequent minimal patterns which are small subgraphs of a regulatory network (Milo et al., 2002). The authors also try to identify the "elementary functions" to which they correspond. We suggest that this method could be extended to minimal feedback circuits. In this case, the method is based on the search for minimal active feedback circuits which determine the dynamic behaviour of a system, and we propose to call it the search for "dynamic similarities". The first step is to find the feedback circuits in the network and determine the relationships within them and between them by extracting minimal graphs from the network. This step, similar to that proposed by Milo et al. (2002), is relatively easy in bacterial transcriptional networks but may require computer assistance for eukarya (Delaplace, 2003). However, feedback circuits are necessary but not sufficient for the existence of "non-trivial" dynamic behaviours such as multistationarity (arising from positive feedback circuits) or homeostasis with or without oscillations (arising from negative feedback circuits).

The second step is to ensure by modelling that the predicted possible behaviour is internally coherent; and this, as we show with the $P$. aeruginosa example, is greatly facilitated by the use of a new software, SMBioNet. This method is based on a theory, temporal logic, usually employed for the logical analysis of the discrete dynamic systems in computer science.

The final, and most time-consuming step is to perform experimental verifications of the hypothesis. This step is fundamental and cannot be dispensed from for several reasons. Not only the existence of feedback circuits in a network does not prove that the corresponding dynamic behaviour actually occurs, but the many simplifications that have lead to the hypothesis are always liable to lead to underestimating the importance of factors externals to the graph. For instance, positive loops can turn negative or vice versa depending upon covalent modification or the interacting partner of one element of these loops. This is why it is so important that experiments to 
validate or refute the epigenetic hypothesis can be designed in a computer-aided manner. This step is necessary to ascertain dynamic similarity from a recognised motif.

Here we have focussed on two potentially "dynamically similar" networks controlling two phenotypes acquired by the same bacterium in the same conditions. Surprisingly, it turns out that the same motif, of an interlocking positive loop and negative circuit, is extremely frequent in quite different networks (Demongeot et al., 2000 ). But the presence of such a motif is only a hint of dynamic similarity, unless the relevant experimental proof is provided.

As explained previously (Guespin-Michel and Kaufman, 2001), if a pathogenic phenotype were to arise through an epigenetic modification instead of a mutation, it would have important implications for therapy since it would make sense to search for drugs that influence the epigenetic switch instead of using antibiotics to which bacteria become resistant (Norris et al., 1999). A systematic search for dynamic "non-trivial" behaviours in bacteria (Guespin-Michel, 2001; Guespin-Michel et al., 2003) may help create a new field of knowledge with new applications. The same holds true for the Eukarya (Demongeot, 1998). Weinstein (2000) wrote that even if the development of microarray methods and proteomics expands our ability to assess complex profiles of gene expression in cancer cells, these methods do not provide the dynamic view of the actual circuitry of these cells. Recently, however, direct experimental evidence of an epigenetic mechanism has been reported: the MYC oncogene is frequently overexpressed in cancer cells and its brief inactivation in an osteogenic sarcoma cell line appeared to cause epigenetic changes that rendered the cells insensitive to $M Y C$ induced tumorigenesis (Jain et al., 2002).

\section{ACKNOWLEDGEMENTS}

We thank Genopole Recherche for supporting this research, which was performed in the context of the Observability group of the Genopole epigenesis programme. We also thank Vic Norris for helpful discussions.

\section{REFERENCES}

Alm, E. and A.P. Arkin (2003). Biological networks. Current Opinion in Structural Biology 13: 193-202.

Banwart, B., M.L. Splaingard, P.M. Farrell, M.J. Rock, P.L. Havens, J. Moss, M.E. Ehrmantraut, D.W. Frank and J.T. Barbieri (2002). Children with cystic fibrosis produce an immune response against exoenzyme S, a type III cytotoxin of Pseudomonas aeruginosa. Journal of Infectious Diseases 185: 269-270.

Bernot, G., J.F. Guespin-Michel, A. Zemerline, J.-P. Comet, P. Amar, F. Delaplace and P. Ballet (2002). Modelling, observability and experiment: a case study? Research Repport No. 82, $\mathrm{ftp} / / / \mathrm{ftp}$.lami.univ-evry.fr/pub/publications/reports/2002/lami_82.pdf, LaMI, Univ. of Evry.

Bernot, G., J.F. Guespin-Michel, J.-P. Comet, P. Amar, A. Zemirline, F. Delaplace, P. Ballet, A. Richard.(2003a). Modelling, observability and experiment: a case study. In: Amar, P., F. Képès, V. Norris and P. Tracqui (Eds). Proceedings of the Dieppe Spring school on Modelling and simulation of biological processes in the context of genomics. pp. 49-55. Frontier Group, Paris.

Bernot, G., J.-P. Comet, A. Richard and J.F. Guespin-Michel (2003b). A Fruitful Application of Formal Methods to Biological Regulatory Networks: extending Thomas' logical approach with temporal logic", Research Report No. 87, LaMI, Univ. of Evry, France. 
$\mathrm{ftp} / / / \mathrm{ftp}$.lami.univ-evry.fr/pub/publications/reports/2003/lami_87.ps

Bernot, G., J.-P. Comet, A. Richard and J.F. Guespin-Michel (2004). A Fruitful Application of Formal Methods to Biological Regulatory Networks: extending Thomas' asynchronous logical approach with temporal logic. Journal of Theoretical Biology (in press).

Boucher, J.C., H.Yu, M. H. Mudd and V. Deretic (1997). Mucoid Pseudomonas aeruginosa in cystic fibrosis: Characterization of muc mutations in clinical isolates and analysis of clearance in a model of respiratopry infection. Infection and Immunity. 65: 3838-3846.

Casadesus, J. and R. D'Ari ( 2002). Memory in bacteria and phage. Bioessays 2: 4512-4518.

Cinquin, O. and J. Demongeot (2002). Positive and negative feedback: striking a balance between necessary antagonists. Journal of Theoretical Biology 216: 229-241.

Dacheux, D., B.Toussaint, M. Richard, G.Brochier, J.Croize, and I. Attree (2000). Pseudomonas aeruginosa cystic fibrosis isolates induce rapid, type III secretion-dependant, but ExoU independent oncosis of macrophages and polymorphonuclear neutrophils. Infection and Immunity 68: 2916-2924.

Dacheux, D., J. Goure, J. Chabert, Y. Usson, and I. Attree (2001a). Pore forming activity of type III system-secreted proteins lead to oncosis of macrophages and polymorphonuclear neutrophils. Molecular Microbiology 40: 76-85.

Dacheux, D., I. Attree and B. Toussaint (2001b). Expression of ExsA in trans confers type III secretion system-dependent cytotoxicity on noncytotoxic Pseudomonas aeruginosa cystic fibrosis isolates. Infection and Immunity 69: 538-542.

Dacheux, D., O. Epaulard, A. De Groot, B. Guery, R. Leberre, I. Attree, B. Polack and B. Toussaint (2002). Activation of the Pseudomonas aeruginosa type III secretion system requires intact pyruvate dehydrogenase ace AB operon. Infection and Immunity 70: 3973-77.

Delaplace, F. (2003). Apport de l'analyse statique à la programmation scientifique. Habilitation à Diriger les Recherches. Université d'Evry, France.

Delbrück, M. (1949). Unités biologiques douées de continuité génétique. Colloques internationaux du CNRS, Paris.

Demongeot, J. (1998). Multistationarity and cell differentiation. Journal of Biological Systems 6: $1-2$.

Demongeot, J., M. Kaufman and R. Thomas. (2000). Positive feedback circuits and memory. Comptes Rendus de l'Académie des Sciences, Paris 323: 69-79.

Feltman, H., G. Schulert, S. Khan, M. Jain, L. Peterson and A. R. Hauser (2001). Prevalence of type III secretion genes in clinical and environmental isolates of Pseudomonas aeruginosa. Microbiology UK 147: 2659-2069.

Franck, D.W. (1997). The exoenzyme S regulon of Pseudomonas aeruginosa. Molecular Microbiology 26: 621-629.

Govan, J. R. W. and G. S. Harris (1986). Pseudomonas aeruginosa and cystic fibrosis: unusual bacterial adaptation and pathogenesis. Microbiological Sciences 3: 302-308.

Guespin-Michel, J.F. (2001). Epigenesis- a request for information on lost of adaptive phenotypes. Microbiology UK 147: 251-252.

Guespin-Michel, J.F. and M. Kaufman (2001). Positive feedback circuits and adaptive regulations in bacteria. Acta Biotheoretica 49: 207 - 218.

Guespin-Michel, J.F., B. Polack and A. Mérieau (2003). Bacterial adaptation and epigenesis. Recent Research Developments in Microbiology 7: 289-305.

Hueck, C.J. (1998). Type III protein secretion systems in bacterial pathogens of animals and plants. Microbiological Molecular Biology Reviews 62: 379-433.

Huth, M. R. and M. D. Ryan (2000). Logic in Computer Science. Modelling and Reasoning about Systems. Cambridge University Press, Cambridge.

Jain, M., C. Arvanitis, K., Chu, W. Dewey, E. Leonhardt, M.Trinh, C. D. Sundberg, J. M. Bishop and D. W. Felsher (2002). Sustained loss of a neoplastic phenotype by brief inactivation of MYC. Science 297: 102-104. 
Kaufman, M., and R. Thomas (2003). Emergence of complex behaviour from simple circuits structures. Comptes Rendus Biologies 326: 205-214.

McCaw, M.L., G.L. Lykken, P.K. Singh and T.L. Yahr (2002). ExsD is a negative regulator of the Pseudomonas aeruginosa type III secretion regulon. Molecular Microbiology 46: $1123-1133$

Miller, V.L. (2002). Connections between transcriptional regulation and type III secretion? Current Opinion in Microbiology 5: 211-215.

Milo, R., S. Shen-Orr, S. Itzkovitz, N. Kashtan, D. Chklovskii and U. Alon (2002). Network motifs: simple building blocks of complex networks. Science 298: 824-827.

Moss, J., M. E. Ehrmantraut, B. D. Banwart, D. W. Frank and J. T. Barbieri (2001). Sera from adult patients with cystic fibrosis contain antibodies to Pseudomonas aeruginosa type III apparatus. Infection and Immunity 69: 118561188.

Norris, V., P. Freestone, S. Grant, I. Toth, M.Trinei, S. C. Clarke, L. Le Sceller, T. Nyström, A. Oshima, and T. Onoda (1999). Tyrosine phosphorylation in E. coli has implications for the manipulation of intra-cellular signalling and structure in anti-bacterial therapies: the Jekyll and Hyde approach. Emerging Therapeutic Targets 3: 89-92.

Pérès, S. and J.-P. Comet (2003). Contribution of computational tree logic to biological regulatory networks: example from Pseudomonas aeruginosa. In: Proceedings of the International workshop on Computational Methods in Systems Biology (CMSB) 2602: 47-56.

Richard, A., J.-P. Comet and G. Bernot, «SMBioNet: Selection of Models of Biological Networks », http://smbionet.lami.univ-evry.fr/

Rowen, D. W., and V. Deretic (2000). Membrane to cytosol redistribution of ECF sigma factor $\mathrm{Alg} \mathrm{U}$ and conversion to Mucoidy in Pseudomonas aeruginosa isolates from cystic fibrosis patients. Molecular Microbiology 36: 314-327.

Schurr, M.J., D.W. Martin, M.H. Mudd and V. Deretic (1994). Gene cluster controlling conversion to alginate-overproducing phenotype in Pseudomonas aeruginosa: functional analysis in a heterologous host and role in the instability of mucoidy. Journal of Bacteriology 176: 3375-3382.

Snoussi, E.H. (1989). Qualitative dynamics of piece-linear differential equations: a discrete mapping approach. Dynamic and Stability of Systems 4: 189-207.

Snoussi, E.H. (1998). Necessary conditions for multistationarity and stable periodicity. Journal of Biological Systems 6: 3-9.

Soulé, C. (2003). Graphic requirements for multistationarity. ComPleXus 1: 123-133.

Thomas, R. (1981). On the relation between the logical structure of systems and their ability to generate multiple steady states or sustained oscillations. In: Dora, D., J. Demongeot and Lacolle (Eds). Numerical Methods in the Study of Critical Phenomena. pp 180-193. Springer-Verlag, Berlin.

Thomas, R. (1998). Laws for the dynamics of regulatory networks. International Journal of Developmental Biology 42: 479-485.

Thomas, R. and D. Thieffry (1995). Les boucles de rétroaction rouages des réseaux de régulation biologiques. Médecine/Science 11: 189-197.

Thomas, R. and M. Kaufman (2001). Multistationarity, the basis of cell differentiation and memory. II. Logical analysis of regulatory networks in terms of feedback circuits. Chaos 11: 180-195.

Thomas, R. and R. D'Ari (1990). Biological feedback. CRC Press, Boca Raton, Florida.

Weinstein, I.B. (2000). Disorders in cell circuitry during multistage carcinogenesis: the role of homeostasis. Carcinogenesis 21: 857-864. 